\title{
Near-to-stoichiometric acidic recovery of spent lithium-ion battery through induced crystallization
}

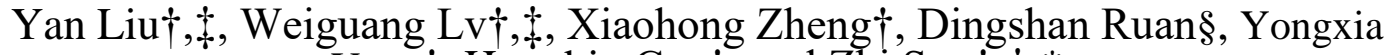
Yang $\dagger$, Hongbin Cao†, and Zhi Sun†, ${ }^{\dagger}, *$

${ }^{\dagger}$ Beijing Engineering Research Center of Process Pollution Control, Institute of Process Engineering, Innovation Academy for Green Manufacture, Chinese Academy of Sciences, Beijing 100190, China

${ }^{\sharp}$ School of Chemical Engineering, University of Chinese Academy of Sciences, Beijing 101407, China

${ }^{8}$ Central South University, Changsha, 314500, China

* Corresponding author. E-mail: sunzhi@ipe.ac.cn, Tel: +86 108254 4844, Fax: +86 108254

4845, Address: NO.1 Beierjie, Zhongguancun, Beijing, China

Column of article: Research

Type of article: Article

Subject: Green Industrial Processes

Supporting Information Content:

Number of pages: 7

Number of figures: 4

Number of tables 11

* Corresponding author. Tel.: +86 108254 4844; fax: +86 1082544845 .

E-mail address: sunzhi@ipe.ac.cn (Z.Sun) 
Results and discussion

Effect of different parameters on the synergistic leaching of NCM523 and LFP

The leaching experiments under different $\mathrm{H}_{2} \mathrm{SO}_{4}$ concentration were conducted under the following conditions: $\mathrm{N} / \mathrm{L}$ mass ratio of $0.5: 1, \mathrm{~S} / \mathrm{L}$ ratio of $32 \mathrm{~g} / \mathrm{L}$, leaching at $80^{\circ} \mathrm{C}$, leaching time of $4 \mathrm{~h}$, seed crystal $5 \mathrm{~g} / \mathrm{L}$ and $\mathrm{H}_{2} \mathrm{SO}_{4}$ concentration ranging from $0.1 \mathrm{~mol} / \mathrm{L}$ to $0.5 \mathrm{~mol} / \mathrm{L}$ respectively. The experimental results are presented in Table S1.

Table S1 Leaching efficiencies of metals under different $\mathrm{H}_{2} \mathrm{SO}_{4}$ concentration

\begin{tabular}{ccccccc}
\hline $\mathrm{H}_{2} \mathrm{SO}_{4}$ concentration & \multicolumn{6}{c}{ Leaching efficiencies (\%) } \\
\cline { 2 - 7 }$(\mathrm{mol} / \mathrm{L})$ & $\mathrm{Co}$ & $\mathrm{Fe}$ & $\mathrm{Li}$ & $\mathrm{Mn}$ & $\mathrm{Ni}$ & $\mathrm{P}$ \\
\hline 0.1 & 9.918889 & 28.67119 & 44.13406 & 9.287721 & 10.23584 & 27.67294 \\
0.2 & 79.81883 & 9.019507 & 98.42443 & 80.69095 & 78.98092 & 7.607095 \\
0.25 & 98.1686 & 1.455008 & 98.5255 & 96.61598 & 98.24867 & 0.631258 \\
0.3 & 99.15538 & 3.063018 & 105.2601 & 99.44233 & 99.22458 & 1.827764 \\
0.4 & 95.739 & 13.3844 & 97.96063 & 95.75188 & 96.55574 & 11.79068 \\
0.5 & 98.92698 & 17.46788 & 99.01009 & 98.77058 & 99.42675 & 16.27459 \\
\hline
\end{tabular}

The leaching experiments under different N/L mass ratio were conducted under the following conditions: $0.25 \mathrm{~mol} / \mathrm{L}$ sulfuric acid, $\mathrm{S} / \mathrm{L}$ ratio of $32 \mathrm{~g} / \mathrm{L}$, leaching at $80^{\circ} \mathrm{C}$, leaching time of $4 \mathrm{~h}$, seed crystal $5 \mathrm{~g} / \mathrm{L}$ and $\mathrm{N} / \mathrm{L}$ mass ratio ranging from 0.2 to 1.2 respectively. The experimental results are presented in Table S2.

Table S2 Leaching efficiencies of metals under different N/L mass ratio

\begin{tabular}{ccccccc}
\hline \multirow{2}{*}{ N/L mass ratio } & \multicolumn{7}{c}{ Leaching efficiencies (\%) } \\
\cline { 2 - 7 } & $\mathrm{Co}$ & $\mathrm{Fe}$ & $\mathrm{Li}$ & $\mathrm{Mn}$ & $\mathrm{Ni}$ & $\mathrm{P}$ \\
\hline 0.2 & 85.09852 & 38.21825 & 96.96093 & 94.95321 & 96.20618 & 37.59199 \\
0.4 & 92.08363 & 9.374078 & 98.08902 & 94.53928 & 95.8067 & 7.588102 \\
0.5 & 98.1686 & 1.455008 & 98.5255 & 96.61598 & 98.24867 & 0.631258 \\
0.6 & 85.91701 & 0.083801 & 97.79252 & 90.97651 & 87.12598 & 0.146815 \\
0.8 & 70.77478 & 0.09241 & 92.88583 & 73.51315 & 70.80134 & 0.544926 \\
1 & 54.91726 & 8.298451 & 83.99513 & 55.3981 & 55.64927 & 6.103031 \\
1.2 & 40.18288 & 29.92783 & 71.85806 & 38.7084 & 41.28839 & 25.69838 \\
\hline
\end{tabular}

The leaching experiments at different leaching time and seed crystal were conducted under the following conditions: $\mathrm{N} / \mathrm{L}$ mass ratio of $0.5: 1,0.25 \mathrm{~mol} / \mathrm{L}$ sulfuric acid, $\mathrm{S} / \mathrm{L}$ ratio of $32 \mathrm{~g} / \mathrm{L}$, leaching at $80^{\circ} \mathrm{C}$, seed crystal from $0 \mathrm{~g} / \mathrm{L}$ to $7 \mathrm{~g} / \mathrm{L}$ and leaching time ranging from $0.5 \mathrm{~h}$ to $4 \mathrm{~h}$ respectively. The experimental results are presented in Table S3-S6.

Table S3 Leaching efficiencies of metals at different leaching time (seed crystal $5 \mathrm{~g} / \mathrm{L}$ )

\begin{tabular}{ccccccc}
\hline Leaching & \multicolumn{5}{c}{ Leaching efficiencies (\%) } \\
\cline { 2 - 7 } time (h) & $\mathrm{Co}$ & $\mathrm{Fe}$ & $\mathrm{Li}$ & $\mathrm{Mn}$ & $\mathrm{Ni}$ & $\mathrm{P}$ \\
\hline 0.5 & 16.7934 & 87.19037 & 69.98913 & 22.1702 & 24.79174 & 78.35728 \\
1 & 28.11203 & 64.68273 & 73.4909 & 27.21843 & 29.69137 & 62.49214 \\
1.5 & 58.34105 & 41.9235 & 83.89825 & 57.20138 & 58.60726 & 39.1194 \\
2 & 68.71907 & 33.04546 & 88.25878 & 67.48864 & 68.30092 & 30.46587 \\
2.5 & 76.8947 & 18.65668 & 92.42837 & 77.04044 & 77.03473 & 17.07654
\end{tabular}




\begin{tabular}{ccccccc}
3 & 87.15818 & 9.73212 & 97.12072 & 87.1159 & 87.19181 & 8.18483 \\
3.5 & 92.86538 & 7.84495 & 98.81721 & 92.0201 & 92.67007 & 6.79572 \\
4 & 96.56193 & 2.95907 & 98.66747 & 96.20068 & 96.47402 & 2.89199 \\
\hline
\end{tabular}

Table S4 Leaching efficiencies of metals at different leaching time (seed crystal 0g/L)

\begin{tabular}{ccccccc}
\hline \multirow{2}{*}{$\begin{array}{c}\text { Leaching } \\
\text { time }(\mathrm{h})\end{array}$} & $\mathrm{Co}$ & $\mathrm{Fe}$ & $\mathrm{Li}$ & $\mathrm{Mn}$ & $\mathrm{Ni}$ & $\mathrm{P}$ \\
\cline { 2 - 7 } & 17.26644 & 81.85804 & 70.61735 & 18.21853 & 20.5287 & 80.35605 \\
0.5 & 21.56062 & 81.36118 & 71.93111 & 20.40741 & 22.95981 & 79.10837 \\
1 & 22.23519 & 77.64133 & 71.14909 & 21.11554 & 23.60851 & 75.5616 \\
1.5 & 21.86537 & 76.30533 & 71.66369 & 20.95777 & 23.29558 & 73.93715 \\
2 & 22.57536 & 75.55767 & 71.97945 & 22.23374 & 24.97924 & 72.24306 \\
2.5 & 23.88223 & 73.99883 & 71.58608 & 23.63338 & 26.35056 & 70.05219 \\
3 & 23.91004 & 70.33246 & 73.3962 & 24.31588 & 26.89576 & 66.14914 \\
3.5 & 25.15889 & 68.33246 & 74.36238 & 24.70833 & 27.0604 & 64.14914 \\
4 & & & & &
\end{tabular}

Table S5 Leaching efficiencies of metals at different leaching time (seed crystal 2g/L)

\begin{tabular}{ccccccc}
\hline \multirow{2}{*}{$\begin{array}{c}\text { Leaching } \\
\text { time }(\mathrm{h})\end{array}$} & $\mathrm{Co}$ & $\mathrm{Fe}$ & $\mathrm{Li}$ & $\mathrm{Mn}$ & $\mathrm{Ni}$ & $\mathrm{P}$ \\
\cline { 2 - 7 } & 16.96107 & 88.29096 & 70.20556 & 21.64529 & 20.07545 & 86.2296 \\
0.5 & 24.23876 & 81.552 & 74.11433 & 21.14821 & 23.9561 & 80.52649 \\
1 & 27.5945 & 78.23679 & 76.15543 & 22.17255 & 26.11481 & 75.29567 \\
1.5 & 31.71744 & 70.06578 & 80.26512 & 26.77659 & 30.83254 & 70.58736 \\
2 & 67.1081 & 56.51139 & 87.62689 & 40.21847 & 44.45278 & 55.08777 \\
2.5 & 76.76437 & 27.41359 & 92.14044 & 70.75472 & 76.2867 & 24.51328 \\
3 & 88.58729 & 11.85026 & 96.34781 & 85.37548 & 92.05191 & 9.12353 \\
3.5 & 91.85095 & 5.67929 & 97.05332 & 91.21253 & 98.24064 & 0.24886 \\
4 & & & & & &
\end{tabular}

Table S6 Leaching efficiencies of metals at different leaching time (seed crystal 7g/L)

\begin{tabular}{ccccccc}
\hline \multirow{2}{*}{$\begin{array}{c}\text { Leaching } \\
\text { time }(\mathrm{h})\end{array}$} & $\mathrm{Co}$ & $\mathrm{Fe}$ & $\mathrm{Li}$ & $\mathrm{Mn}$ & $\mathrm{Ni}$ & $\mathrm{P}$ \\
\cline { 2 - 7 } & 22.17006 & 82.10961 & 69.33072 & 22.1702 & 24.79174 & 76.15242 \\
0.5 & 27.3901 & 54.47628 & 81.23957 & 27.46929 & 30.1757 & 56.58985 \\
1 & 49.55833 & 33.20582 & 88.03447 & 49.98496 & 51.89551 & 37.14327 \\
1.5 & 87.61938 & 27.0696 & 98.00448 & 88.13131 & 89.99263 & 24.55513 \\
2 & 90.53 & 16.60672 & 98.35869 & 91.84691 & 93.83035 & 15.06754 \\
2.5 & 94.55 & 9.67 & 98.88643 & 96.50508 & 99.06584 & 8.25924 \\
3 & 96.68 & 8.68645 & 97.36403 & 97.11307 & 99.06584 & 6.60695 \\
3.5 & 97.56292 & 6.09366 & 98.46811 & 98.19087 & 99.06584 & 4.22703 \\
4 & & & & & &
\end{tabular}

The leaching experiments at different temperature were conducted under the following conditions: $\mathrm{N} / \mathrm{L}$ mass ratio of $0.5: 1,0.25 \mathrm{~mol} / \mathrm{L}$ sulfuric acid, $\mathrm{S} / \mathrm{L}$ ratio of $32 \mathrm{~g} / \mathrm{L}$, leaching time of $4 \mathrm{~h}$, seed crystal $5 \mathrm{~g} / \mathrm{L}$ and temperature ranging from $20^{\circ} \mathrm{C}$ to $80^{\circ} \mathrm{C}$ respectively. The experimental results are presented in Table S7. 
Table S7 Leaching efficiencies of metals at different temperature

\begin{tabular}{ccccccc}
\hline Temperature & \multicolumn{5}{c}{ Leaching efficiencies (\%) } \\
\cline { 2 - 7 }$\left({ }^{\circ} \mathrm{C}\right)$ & $\mathrm{Co}$ & $\mathrm{Fe}$ & $\mathrm{Li}$ & $\mathrm{Mn}$ & $\mathrm{Ni}$ & $\mathrm{P}$ \\
\hline 20 & 50.64378 & 75.0847 & 81.96568 & 49.83763 & 52.83847 & 71.83612 \\
40 & 40.55418 & 65.9502 & 78.48987 & 39.47527 & 42.75286 & 61.82354 \\
60 & 44.68366 & 53.83658 & 87.88777 & 42.52302 & 45.07048 & 48.45042 \\
80 & 98.1686 & 1.455008 & 98.5255 & 96.61598 & 98.24867 & 0.631258 \\
\hline
\end{tabular}

Leaching Mechanism.

Thermodynamic data of species in $\mathrm{Li}-\mathrm{Fe}-\mathrm{P}-\mathrm{H}_{2} \mathrm{O}$ system at $298.15 \mathrm{~K}$ was shown in Table S8. The reactions and corresponding E-pH equations were presented in Table S9. The E-pH diagram has been calculated at the concentration of $[\mathrm{Li}]=[\mathrm{Fe}]=[\mathrm{P}]=1 \mathrm{~mol} \cdot \mathrm{L}^{-1}$.

Table S8 Thermodynamic data of species in Li-Fe-P-H2O system at $298.15 \mathrm{~K}^{1,2}$

\begin{tabular}{|c|c|c|c|c|c|}
\hline Species & $\Delta_{\mathrm{f}} \mathrm{G}^{\theta}(\mathrm{kJ} / \mathrm{mol})$ & Species & $\Delta_{\mathrm{f}} \mathrm{G}^{\theta}(\mathrm{kJ} / \mathrm{mol})$ & Species & $\Delta_{\mathrm{f}} \mathrm{G}^{\theta}(\mathrm{kJ} / \mathrm{mol})$ \\
\hline $\mathrm{LiFePO}_{4}$ & -1481.0 & $\mathrm{FePO}_{4}$ & -1182.23 & $\mathrm{Li}_{3} \mathrm{PO}_{4}$ & -1965.90 \\
\hline $\mathrm{PO}_{4}^{3-}$ & -1025.49 & $\mathrm{Li}^{+}$ & -292.90 & $\mathrm{H}_{3} \mathrm{PO}_{4}$ & -1118.92 \\
\hline $\mathrm{Fe}^{2+}$ & -91.53 & $\mathrm{Fe}^{3+}$ & -17.18 & $\mathrm{Fe}(\mathrm{OH})_{2}$ & -491.95 \\
\hline $\mathrm{Fe}(\mathrm{OH})_{3}$ & -705.58 & $\mathrm{HPO}_{4}^{2-}$ & -1095.99 & $\mathrm{H}_{2} \mathrm{PO}_{4}{ }^{-}$ & -1137.15 \\
\hline $\mathrm{H}_{2} \mathrm{O}$ & -237.14 & & & & \\
\hline
\end{tabular}

Table S9 Equilibrium equations relative to the E-pH diagram of $\mathrm{Li}-\mathrm{Fe}-\mathrm{P}-\mathrm{H}_{2} \mathrm{O}$ system

\begin{tabular}{|c|c|c|}
\hline No. & Reactions & E-pH equations \\
\hline 1 & $\mathrm{Fe}^{3+}+\mathrm{e}=\mathrm{Fe}^{2+}$ & $\mathrm{E}=0.770-0.0592 \lg \left[\mathrm{Fe}^{2+}\right] /\left[\mathrm{Fe}^{3+}\right]$ \\
\hline 2 & $\mathrm{FePO}_{4}+3 \mathrm{H}^{+}=\mathrm{Fe}^{3+}+\mathrm{H}_{3} \mathrm{PO}_{4}+2 \mathrm{H}_{2} \mathrm{O}$ & $\mathrm{pH}=-2.69-1 / 3 \lg \left[\mathrm{Fe}^{3+}\right]\left[\mathrm{H}_{3} \mathrm{PO}_{4}\right]$ \\
\hline 3 & $\mathrm{FePO}_{4}+3 \mathrm{H}^{+}+\mathrm{e}=\mathrm{Fe}^{2+}+\mathrm{H}_{3} \mathrm{PO}_{4}$ & $\begin{array}{c}\mathrm{E}=0.1504-0.0592 \lg \left[\mathrm{Fe}^{2+}\right]\left[\mathrm{H}_{3} \mathrm{PO}_{4}\right]- \\
0.1775 \mathrm{pH}\end{array}$ \\
\hline 4 & $\mathrm{LiFePO}_{4}+3 \mathrm{H}^{+}=\mathrm{Fe}^{2+}+\mathrm{Li}^{+}+\mathrm{H}_{3} \mathrm{PO}_{4}$ & $\mathrm{pH}=1.355-1 / 3 \lg \left[\mathrm{Li}^{+}\right]\left[\mathrm{Fe}^{2+}\right]\left[\mathrm{H}_{3} \mathrm{PO}_{4}\right]$ \\
\hline 5 & $\mathrm{FePO}_{4}+\mathrm{Li}^{+}+\mathrm{e}=\mathrm{LiFePO}_{4}$ & $\mathrm{E}=0.04145+0.0592 \lg \left[\mathrm{Li}^{+}\right]$ \\
\hline 6 & $\begin{array}{c}\mathrm{Li}_{3} \mathrm{PO}_{4}+\mathrm{Fe}(\mathrm{OH})_{3}+3 \mathrm{H}^{+}=\mathrm{FePO}_{4}+3 \mathrm{Li}^{+}+ \\
3 \mathrm{H}_{2} \mathrm{O}\end{array}$ & $\mathrm{pH}=6.098-\lg \left[\mathrm{Li}^{+}\right]$ \\
\hline 7 & $\begin{array}{c}\mathrm{Fe}(\mathrm{OH})_{3}+\mathrm{Li}_{3} \mathrm{PO}_{4}+3 \mathrm{H}^{+}+\mathrm{e}=\mathrm{LiFePO}_{4}+ \\
2 \mathrm{Li}^{+}+3 \mathrm{H}_{2} \mathrm{O}\end{array}$ & $\begin{array}{c}\mathrm{E}=1.1239-0.1183 \lg \left[\mathrm{Li}^{+}\right]- \\
0.1775 \mathrm{pH}\end{array}$ \\
\hline 8 & $\begin{array}{c}\mathrm{Fe}(\mathrm{OH})_{2}+\mathrm{Li}_{3} \mathrm{PO}_{4}+2 \mathrm{H}^{+}=\mathrm{LiFePO}_{4}+ \\
2 \mathrm{H}_{2} \mathrm{O}+2 \mathrm{Li}^{+}\end{array}$ & $\mathrm{pH}=7.4253-\lg \left[\mathrm{Li}^{+}\right]$ \\
\hline 9 & $\mathrm{Fe}(\mathrm{OH})_{3}+\mathrm{H}^{+}+\mathrm{e}=\mathrm{Fe}(\mathrm{OH})_{2}+\mathrm{H}_{2} \mathrm{O}$ & $\mathrm{E}=0.2452-0.0592 \mathrm{pH}$ \\
\hline 10 & $\mathrm{Fe}(\mathrm{OH})_{2}+2 \mathrm{H}^{+}=\mathrm{Fe}^{2+}+2 \mathrm{H}_{2} \mathrm{O}$ & $\mathrm{pH}=5.3608-1 / 2 \lg \left[\mathrm{Fe}^{2+}\right]$ \\
\hline 11 & $\mathrm{Fe}(\mathrm{OH})_{3}+3 \mathrm{H}^{+}=\mathrm{Fe}^{3+}+3 \mathrm{H}_{2} \mathrm{O}$ & $\mathrm{pH}=0.6192-1 / 3 \lg \left[\mathrm{Fe}^{3+}\right]$ \\
\hline 12 & $\mathrm{Li}_{3} \mathrm{PO}_{4}+3 \mathrm{H}^{+}=\mathrm{H}_{3} \mathrm{PO}_{4}+3 \mathrm{Li}^{+}$ & $\mathrm{pH}=1.9901-\lg \left[\mathrm{Li}^{+}\right]-1 / 3 \lg \left[\mathrm{H}_{3} \mathrm{PO}_{4}\right]$ \\
\hline 13 & $\mathrm{Li}_{3} \mathrm{PO}_{4}+2 \mathrm{H}^{+}=\mathrm{H} 2 \mathrm{PO}_{4}^{-}+3 \mathrm{Li}^{+}$ & $\mathrm{pH}=4.5822-3 / 2 \lg \left[\mathrm{Li}^{+}\right]-1 / 2 \lg \left[\mathrm{H}_{2} \mathrm{PO}_{4}^{-}\right]$ \\
\hline 14 & $\mathrm{Li}_{3} \mathrm{PO}_{4}+\mathrm{H}^{+}=\mathrm{Li}_{2} \mathrm{HPO}_{4}+\mathrm{Li}^{+}$ & $\mathrm{pH}=4.9863-\lg \left[\mathrm{Li}^{+}\right]$ \\
\hline 15 & $\mathrm{FePO}_{4}+2 \mathrm{H}^{+}=\mathrm{Fe}^{3+}+\mathrm{H}_{2} \mathrm{PO}_{4}^{-}$ & $\mathrm{pH}=-3.636-1 / 2 \lg \left[\mathrm{Fe}^{3+}\right]\left[\mathrm{H}_{2} \mathrm{PO}_{4}^{-}\right]$ \\
\hline
\end{tabular}




\begin{tabular}{|c|c|c|}
\hline 16 & $\mathrm{FePO}_{4}+2 \mathrm{H}^{+}+\mathrm{e}=\mathrm{Fe}^{2+}+\mathrm{H}_{2} \mathrm{PO}_{4}{ }^{-}$ & $\begin{array}{c}\mathrm{E}=0.3394-0.0592 \lg \left[\mathrm{Fe}^{2+}\right]\left[\mathrm{H}_{2} \mathrm{PO}_{4}{ }^{-}\right] \\
-0.1183 \mathrm{pH}\end{array}$ \\
\hline 17 & $\mathrm{Fe}(\mathrm{OH})_{3}+2 \mathrm{H}^{+}+\mathrm{HPO}_{4}{ }^{2-}=\mathrm{FePO}_{4}+3 \mathrm{H}_{2} \mathrm{O}$ & $\mathrm{pH}=8.771+0.5 \lg \left[\mathrm{HPO}_{4}{ }^{2-}\right]$ \\
\hline 18 & $2 \mathrm{H}^{+}+2 \mathrm{e}=\mathrm{H}_{2}$ & $\mathrm{E}=-0.0592 \mathrm{pH}$ \\
\hline 19 & $\mathrm{O}_{2}+4 \mathrm{e}+4 \mathrm{H}^{+}=2 \mathrm{H}_{2} \mathrm{O}$ & $\mathrm{E}=1.229-0.0592 \mathrm{pH}$ \\
\hline
\end{tabular}

The leaching experiments at different time were conducted under the following conditions: $\mathrm{N} / \mathrm{L}$ mass ratio of $0.5: 1,0.25 \mathrm{~mol} / \mathrm{L}$ sulfuric acid, $\mathrm{S} / \mathrm{L}$ ratio of $32 \mathrm{~g} / \mathrm{L}$, seed crystal $5 \mathrm{~g} / \mathrm{L}$ and temperature of $80^{\circ} \mathrm{C}$. The SEM micrographs of leaching residues at different time are shown in Figure $\mathrm{S} 1$.
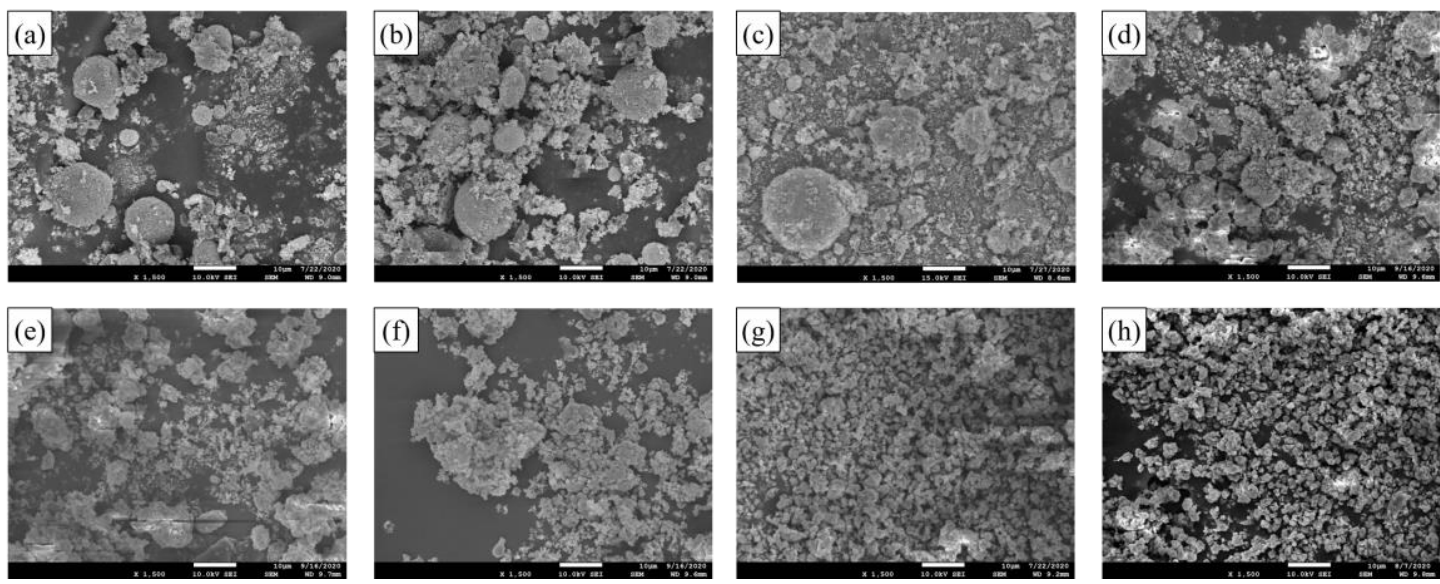

Figure S1 SEM micrograph of leaching residues (NCM523 to LFP mass ratio of $0.5,0.25 \mathrm{~mol} / \mathrm{L} \mathrm{H}_{2} \mathrm{SO}_{4}$, $\mathrm{T}=80^{\circ} \mathrm{C}$ ) at (a) $0.5 \mathrm{~h},(\mathrm{~b}) 1 \mathrm{~h},(\mathrm{c}) 1.5 \mathrm{~h},(\mathrm{~d}) 2 \mathrm{~h},(\mathrm{e}) 2.5 \mathrm{~h},(\mathrm{f}) 3 \mathrm{~h},(\mathrm{~g}) 3.5 \mathrm{~h},(\mathrm{~h}) 4 \mathrm{~h}$

Development of a recovery Process for Spent NCM523 and $\mathrm{LiFePO}_{4}$ Batteries

1)Recovery of $\mathrm{Ni}$, Co and Mn by precipitation

Valuable metals $(\mathrm{Ni}, \mathrm{Co}, \mathrm{Mn})$ were recovered as $\mathrm{Ni}-\mathrm{Co}-\mathrm{Mn}$ hydroxide precursor preparation, and lithium carbonate was precipitated. The solution was pumped into the reactor with the speed of $2 \mathrm{~mL} / \mathrm{min}$ and $\mathrm{NaOH}$ solution $(5 \mathrm{M})$ were pumped into at the same speed. The process was conducted under the protection of nitrogen at $65^{\circ} \mathrm{C}$ with stirring speed of 1000 $\mathrm{rpm}$. The $\mathrm{pH}$ should be adjusted and remain at 11 for $24 \mathrm{~h}$. After vacuum filtration and washed with ultrapure water, $\mathrm{Ni}, \mathrm{Co}$, and $\mathrm{Mn}$ hydroxide precipitate was recovered and then dried in a vacuum oven at $80{ }^{\circ} \mathrm{C}$ for $10 \mathrm{~h}$. the SEM micrograph of Ni-Co-Mn hydroxide precursor is shown in Figure $\mathrm{S} 2$

\section{2)Recovery of lithium carbonate}

Saturated $\mathrm{Na}_{2} \mathrm{CO}_{3}$ of stoichiometric amount was added into the raffinate at $20-60{ }^{\circ} \mathrm{C}$ with a speed of $1-5 \mathrm{~mL} / \mathrm{min}$ after the $\mathrm{Ph}$ was adjust to neutral. $\mathrm{The}^{2} \mathrm{Li}_{2} \mathrm{CO}_{3}$ was washed with ultrapure water and dried in a vacuum oven for $12 \mathrm{~h}$. Mass fraction of metals in the $\mathrm{Li}_{2} \mathrm{CO}_{3}$ precipitation was shown in Table S10 and Mass fraction of elements in the $\mathrm{FePO}_{4} \cdot 2\left(\mathrm{H}_{2} \mathrm{O}\right)$ residues was shown in Table S11. 




Figure S2 SEM images of Ni-Co-Mn hydroxide precursor

Table S10 Mass fraction of metals in the $\mathrm{Li}_{2} \mathrm{CO}_{3}$ precipitation

\begin{tabular}{cccccc}
\hline Materials & $\mathrm{Li}_{2} \mathrm{CO}_{3}$ & $\mathrm{Ni}$ & $\mathrm{Co}$ & $\mathrm{Mn}$ & $\mathrm{Fe}$ \\
\hline Content(wt.\%) & 99.93 & 0.0002 & 0.0002 & 0.0002 & 0.0008 \\
\hline
\end{tabular}

Table S11 Mass fraction of elements in the $\mathrm{FePO}_{4} \cdot 2\left(\mathrm{H}_{2} \mathrm{O}\right)$ residues

\begin{tabular}{ccccccc}
\hline Elements & $\mathrm{Li}$ & $\mathrm{Ni}$ & $\mathrm{Co}$ & $\mathrm{Mn}$ & $\mathrm{Fe}$ & $\mathrm{P}$ \\
\hline Content(wt.\%) & 0.095 & 0.197 & 0.078 & 0.197 & 26.15 & 15.85 \\
\hline
\end{tabular}

3)Reuse of leaching residues as seed crystal

The experiments of $\mathrm{FePO}_{4} \cdot 2 \mathrm{H}_{2} \mathrm{O}$ residues were recycled as seed crystals under the conditions: NCM523-to-LFP mass ratio of $0.5: 1$, solid-to-liquid ratio of $32 \mathrm{~g} / \mathrm{L}$, reaction time of $4 \mathrm{~h}$, seed crystal of $5 \mathrm{~g} / \mathrm{L}$ and reaction temperature of $80^{\circ} \mathrm{C}$. The results of seed crystals and leaching residues are compared in Figure S3.

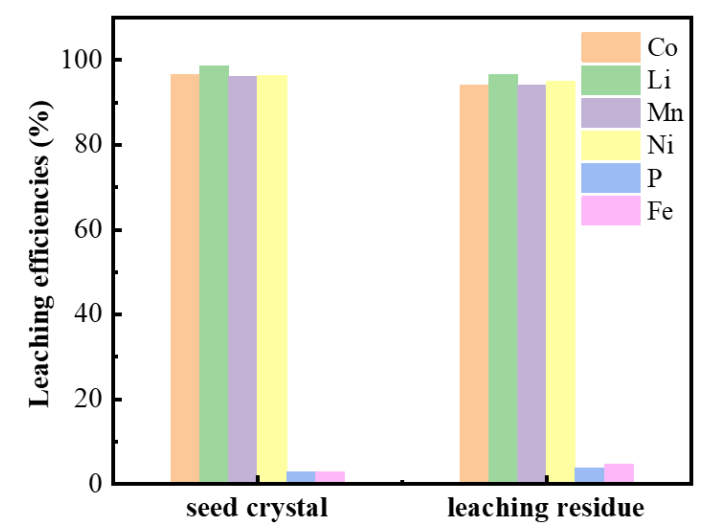

Figure S3 The leaching efficiencies of Li, Co, Mn, Ni, Fe, P from NCM523 using seed crystal (5g/L) 
and leaching residue $(5 \mathrm{~g} / \mathrm{L})$

\section{4)Leaching efficiencies of different types of NCM cathode waste}

The experiments for other types of NCM cathode waste (NCM111, NCM622) were conducted under the same conditions as NCM523: NCM523-to-LFP mass ratio of 0.5:1, solidto-liquid ratio of $32 \mathrm{~g} / \mathrm{L}$, reaction time of $4 \mathrm{~h}$, seed crystal of $5 \mathrm{~g} / \mathrm{L}$ and reaction temperature of $80^{\circ} \mathrm{C}$, and the results of these experiments are presented in Figure S4.

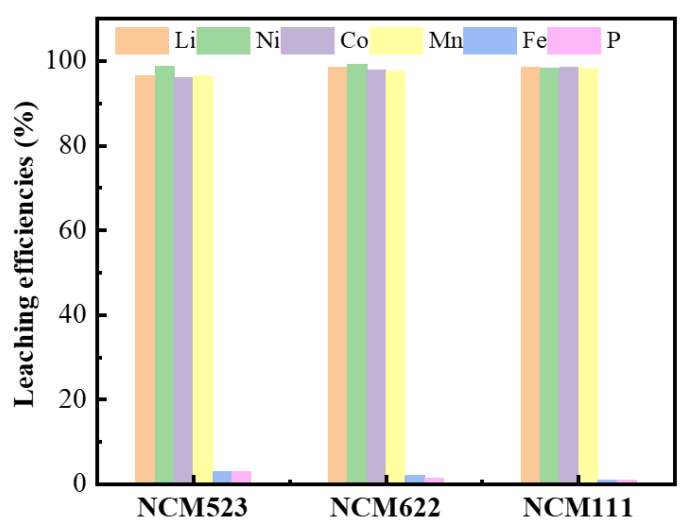

Figure S4 The leaching efficiencies of Li, Co, Mn, Ni, Fe, P from NCM523, NCM622, NCM111, under the leaching conditions of $0.25 \mathrm{~mol} / \mathrm{L} \mathrm{H}_{2} \mathrm{SO}_{4}, \mathrm{~S} / \mathrm{L}=32 \mathrm{~g} \cdot \mathrm{L}^{-1}$, seed crystal $=5 \mathrm{~g} / \mathrm{L}, 80^{\circ} \mathrm{C}$ and $4 \mathrm{~h}$.

\section{References}

(1) Churikov, A. V.; Ivanishchev, A. V.; Ushakov, A. V.; Gamayunova, I. M.; Leenson, I. A. Thermodynamics of $\mathrm{LiFePO}_{4}$ Solid-Phase Synthesis Using Iron(II) Oxalate and Ammonium Dihydrophosphate as Precursors. Journal of Chemical \& Engineering Data. 2013, 58 (6), 1747-1759, DOI:10.1021/je400183k

(2) Zhang, J.; Hu, J.; Liu, Y.; Jing, Q.; Yang, C.; Chen, Y.; Wang, C. Sustainable and Facile Method for the Selective Recovery of Lithium from Cathode Scrap of Spent LiFePO4 Batteries. ACS Sustainable Chemistry \& Engineering. 2019, 7 (6), 5626-5631, DOI:10.1021/acssuschemeng.9b00404 